\title{
Is Histidine promising in alleviating the risk of Metabolic disorder?
}

\section{Abstract \\ Introduction}

In recent times, evidence from published reports; randomized clinical trial (RCT), human case-controls, metabolomics, animal and cell studies is gaining rapt significance on the ameliorative potential of histidine (His) in obesity/insulin resistance (IR)/ inflammation outcomes. This study aimed at systematically mining evidence from previously published studies on the vitality of His on metabolic disorder outcomes.

\section{Methods}

Using the MOOSE guidelines we searched PubMed and Cochrane library using the search words "histidine" and "obesity" or "diabetes" or "metabolic syndrome" and included articles on His intake/administration and obesity/diabetes/metabolic disorders involving human and/or animal experimentation.

\section{Results}

We found low circulating His profile has been associated with metabolic disorders independent of ethnicity [1-4]. Similarly, two separate animal trials [5-6] found His to be significantly lower in diabetic Balb/cA mice and lean Zucker rat. Also, the endogenous absence of His disrupts mitochondrial membrane integrity thereby impairing energy production [7]. In addition, epidemiological report revealed higher dietary His was associated with lower overweight and obesity risk [8]. Though epidemiological reports from the diverse population to assert this observation are insufficient, reports from animal models [9-10] revealed similar conclusions. Also, some reports [11-12] have demonstrated the dose-dependent inhibitory effect of His on some inflammatory markers by assuaging the palmitic acid induced pAkt/Akt expression [12]. Also, His inhibits the up-production of free radicals to impede the extracellular signal-regulated kinase in neurons [13].

\section{Conclusion}

These evidences are novel but primarily limited to mostly obesity. In our opinion, future multi-ethnic longitudinal cohort and trials should consider exploring effects of His on non-fatty alcoholic liver diseases and cardiovascular complications. His might as well be beneficial potentially offering significant hope and non-invasive alternatives in the management of metabolic disorders.

Note: Complete citations available in MEDLINE;

[1] PMID: 20834187 [2] PMID: 22266733 [3] PMID: 21996294 [4] PMID: 23697717 [5] PMID:15878720 [6] PMID:15389298 [7] PMID: 4621606 [8] PMID: 27409634 [9] PMID: 17372311 [10] PMID: 15561489 [11] PMID: 16107255 [12] PMID: 23361591 [13] PMID: 22101981

\section{Conflict of Interest}

There is no conflict of interest 